\title{
Ecological Security Assessment Based on Remote Sensing and Landscape Ecology Model
}

\author{
Pei Liu $\mathbb{D}^{1,2,3}$ Xiaoying Zhang $\mathbb{D}^{2}{ }^{2}$ Chao Ma $\mathbb{D}^{1,2}$ Hebing Zhang, ${ }^{1,2}$ Ruimei Han $\mathbb{D}^{1,2}$ \\ and Xiaofeng $\mathrm{Lu}^{1}$ \\ ${ }^{1}$ Key Laboratory of Spatio-temporal Information and Ecological Restoration of Mines (MNR), Henan Polytechnic University, Jiaozuo, \\ Henan 454003, China \\ ${ }^{2}$ School of Surveying and Mapping Land Information Engineering, Henan Polytechnic University, Jiaozuo, 454003 Henan, China \\ ${ }^{3}$ Collaborative Innovation Center of Aerospace Remote Sensing Information Processing and Application of Hebei Province, China
}

Correspondence should be addressed to Ruimei Han; hrm@hpu.edu.cn

Received 23 October 2020; Accepted 1 June 2021; Published 30 June 2021

Academic Editor: Zhenxing Zhang

Copyright (C) 2021 Pei Liu et al. This is an open access article distributed under the Creative Commons Attribution License, which permits unrestricted use, distribution, and reproduction in any medium, provided the original work is properly cited.

The various ecological processes of human beings are not only restricted by the landscape pattern on the regional scale but also affect the local and regional landscape together with global climate change. To date, most of the research on ecological security is based on the pressure-state-response (PSR) model, while there were a few studies based on the landscape ecology model approach. In addition, there has been little literature focus on the dynamic change process of ecological security, especially the simulation and prediction of the future development trend of ecological security. The purpose of this research is to establish a landscape ecological security evaluation method based on grid division, be aimed at breaking the inherent drawbacks of the administrative region as a unit mode approach, anticipated to better reflect the landscape ecological security status of the study area. A complex framework was constructed by integrating random forest algorithm, Fishnet model, landscape ecology model, and CA-Markov model. Multitemporal remote sensing data were selected as a data source, and land use maps of the study area were obtained through the random forest machine learning algorithm firstly. And then, the study area is divided into 307 grids of $2 \mathrm{~km} \times 2 \mathrm{~km}$ using the Fishnet model. Next, the landscape disturbance index, landscape vulnerability index, and landscape loss index are used on the grid scale to establish a landscape ecological security evaluation model. Finally, ecological security assessment of Zhengzhou city was carried out, and the distribution map of the landscape ecological status in 1986, 1996, 2006, 2016, and predicted for 2026 was obtained. The results of the study showed that, as time goes by, the areas with high ecological safety gradually decrease. It is predicted that by 2026 , the ecological security level of Zhengzhou will be dominated by lower ecological security areas. The research results can provide basic information and decision support for government agencies and land use planners to ensure responsible and sustainable development of the urban environment and ecology.

\section{Introduction}

Landscape ecology evaluates the ecological security status of a certain area through the landscape index and landscape pattern, which can provide hierarchical and integrated information data for the multiscale study of ecosystem functions [1]. Landscape pattern is the key content of landscape ecology research [2]. Landscape pattern and its corresponding changes are a comprehensive reflection of the ecological environment in a certain area due to the interaction of vari- ous factors such as natural and human factors. At the meantime, landscape pattern affects the regional ecological process [3] as well for the type, shape, size, quantity, and spatial combination of landscape patches are the interaction of various disturbing factors. Methods of landscape pattern index, which can reflect the composition and characteristics of the landscape in the spatial structure, can be used to quantitatively describe the change of landscape pattern and spatial heterogeneity [4]. Particularly, changes in landscape pattern will affect the ecological environment to a certain extent, 
thereby affecting ecological security. Researches on regional landscape pattern can effectively reveal ecological status and its corresponding spatial changes. Constructing an ecological security evaluation and analytics model by quantitative evaluation of regional landscape ecological index has certain advantages in the research of regional ecosystem security $[5,6]$.

In the past decades, more attentions have been given to ecological security assessment, and a variety of methods have been developed to evaluate ecological security, such as ecological footprint $[7,8]$, PSR models [9-11], and landscape ecology models [12]; for example, Sun et al. [13] used the PSR model combined methods of AHP and FCE to assess coastal wetland ecosystem health. Yang et al. [8] further improved the ecological footprint method to evaluate ecological security, and Peng et al. [14] took Liaoyuan City as a research case to discuss the possible ecological risks of landscapes at the county scale and the resulting ecological losses. Although different ecological safety assessment methods have different focuses, most of them are based on the PSR model. While the PSR model is applicable to regional ecological evaluations with small spatial scales with a few spatial variabilities and a few influencing factors, it is not applicable to natural disasters where human activities exceed the carrying capacity of the natural environment, and it is impossible to determine ecological security risks and uncertain threats. In addition, the logical connection between pressure and state is not inevitable; it is affected by a variety of complex factors. And the PSR evaluation system overemphasizes the impact of human activities on the ecological environment, and the evaluation results are somewhat one-sided [15]. Compared with the PSR model, the landscape ecology model starts from the ecosystem itself and is based on the structure, function, landscape patch dynamics and ecological succession of the landscape, the integrity and stability of the ecosystem, anti-interference ability, and resilience; the establishment of an evaluation index system can better reflect the temporal and spatial changes of landscape patterns. The landscape ecology model is not only suitable for larger spatial scale landscape design and land use planning but also suitable for smaller scale ecological evaluation [16].

In this research, we propose an improved landscape ecology ecological security evaluation model which can break through the long-term use of administrative regions as the research unit for data collection and analysis calculation $[17,18]$ and can better reflect the landscape ecological security status of the research area [19]. For this purpose, multitemporal remote sensing images are used as a major data source, and a random forest algorithm machine learning algorithm is used to interpret basic information of ecological security evaluation. Next, the landscape disturbance index, vulnerability index, and loss index are integrated to establish the landscape safety index. Then, multitemporal ecological security distribution maps are obtained. Finally, with the help of the CA-Markov model, ecological security trend in future is simulated and predicted. We test the method in Zhengzhou urban area, the core city of Chinese urban concentration. The innovation of this method is as follows: (1) all the indicators used in the research are readily calculated from remote sensing satellite images; (2) the research obtained spatial distribution status of ecological security as well as the dynamic changes of ecological security; (3) simulate and predict the future development trend of ecological security; (4) break the solid-state model of data collection, analysis calculation based on administrative regions as research units in previous research.

The results will be significant to guide regional ecological planning, protection, and development and will be helpful to optimize the allocation of resources, to control the further deterioration of the ecology, and to realize regional sustainable development services [20]. The rest part of the paper is organized as follows: a brief introduction about research area, preliminary work, and preprocessing of datasets is given in Section 2. The proposed research framework based on remote sensing (RS) is drawn and applied to the research area in Section 3. The results are shown in Section 4, and analysis and discussion are given in Section 5. Finally, the conclusions are drawn in Section 6.

\section{Study Area and Datasets}

2.1. The General Situation of the Study Area. The study site is located in Zhengzhou city (Figure 1), the capital of Henan Province in the central part of the P.R. China, with a total area of $7507 \mathrm{~km}^{2}$ as well as a population of 9878000 inhabitants. Zhengzhou is one of the National Central Cities in China and serves as the political, economic, technological, and educational center of the province, as well as a major transportation hub in China. The annual average temperature of the city is $14.5^{\circ} \mathrm{C}$, and the general terrain trend is tilt from southwest to northeast. The study area is undergoing the accelerating of Chinese agglomeration, economic development, and urban expansion.

Zhengzhou city core area is located in the southern part of the North China Plain, the lower reaches of the Yellow River, and the north of central Henan Province, between $112^{\circ} 42^{\prime}-114^{\circ} 14^{\prime}$ east longitude and $34^{\circ} 16^{\prime}-34^{\circ} 58^{\prime}$ north latitude. The city is $135-143 \mathrm{~km}$ long from east to west. The north-south width is $70-78 \mathrm{~km}$, and the total area of the city is about $7446 \mathrm{~km}^{2}$. It has jurisdiction over 6 districts and 5 cities, 2 national-level development zones, and one national-level export processing zone. Zhengzhou city belongs to the warm temperate continental monsoon climate with four distinct seasons: spring, dry and drought; summers are hot, concentrated rainfall; autumn is cool, time is short; long winter, dry and cold, rain and snow rarely. The annual average temperature is $15.6^{\circ} \mathrm{C}$, July is the hottest, and the monthly average temperature is $27.3^{\circ} \mathrm{C}$. January is the coldest month, with an average monthly temperature of $-0.2^{\circ} \mathrm{C}$, and most of the rainfall is concentrated in July, August, and September. This paper selects the urban area of Zhengzhou city, including Huiji District, Jinshui District, Zhongyuan District, Guancheng District, and Erqi District as the study area (Figure 1).

2.2. Data Sources and Preprocessing. The selected datasets include multitemporal Landsat remote sensing images of Zhengzhou city captured on April 23, 1986, April 20, 1996, April 30, 2006, and May 11, 2016, from USGS. Images on 


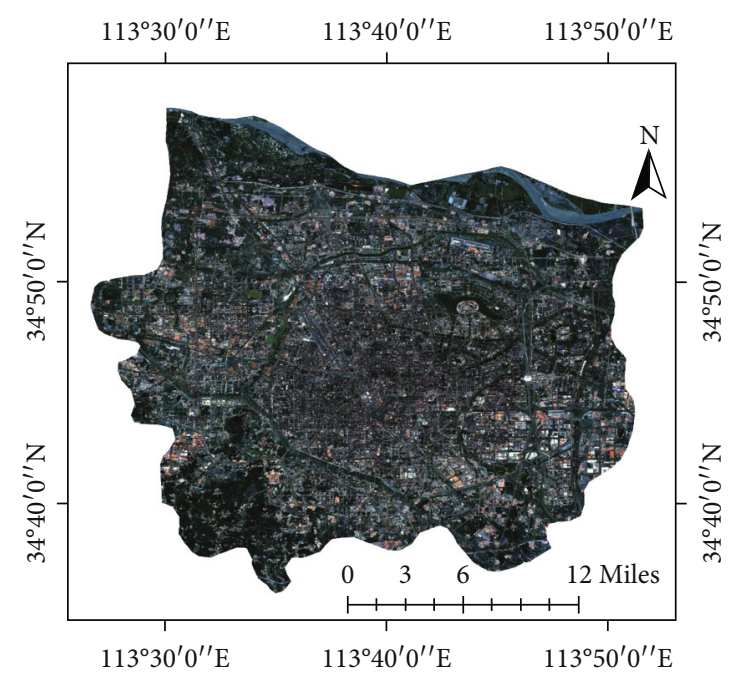

Figure 1: Location of Zhengzhou city, Henan Province, China.

April 23, 1986, April 20, 1996, and April 30, 2006, were obtained with Landsat5 TM sensor; while the image on May 11, 2016, was obtained with Landsat8 OLI sensor. The preprocessing of selected datasets includes radiation calibration, atmospheric correction, and geometric correction.

\section{Methodology}

3.1. Random Forest and Accuracy Assessment. Random forest $(\mathrm{RF})$ is a state-of-art machine learning algorithm proposed by Leo Breiman and Adele Cutler in 2001 that combined bagging ensemble learning theory with a random subspace method [21-23]. The prediction result of the random forest algorithm is obtained by solving the mode of the decision results of multiple decision trees that make up the forest. Each time, about $2 / 3$ of the samples from the original training dataset were extracted with bootstrap sampling technology for decision tree model training in the corresponding epoch. So some samples in the original training set may be used to perform the decision tree at the same time. The remaining about $1 / 3$ of the samples are drawn for the out-of-bag error evaluation of the random forest model, and then, the prediction error rate of the model is calculated [24]. The basic idea of random forest classification $[21,25]$ can be described as three steps: (1) extracting $k$ samples from the original training set using the bootstrap method, and ensuring the percentage of each class in subsets is the same as the original training set; (2) building $k$ decision tree models for $k$ sample subsets to obtain $k$ classification results; (3) voting all classification results to obtain a final classified map. The two most important parameters in these procedures are number of trees used to construct RF and number of features selected for each individual tree. The second parameter is much more important than the first one by literature review [26-28]. In this research, the number of features is setting as $\log 2 d$, where $d$ is all features available in this research.

The evaluation of land use classification accuracy is a test of the classification results. There are two commonly used methods. One is to compare the classification results with the actual land use classification data; the other is to use high-resolution remote sensing images to select a certain proportion of training samples to test the accuracy of the classification data. At present, the most commonly used method of classification accuracy evaluation is the confusion matrix method, which is an error matrix, which is expressed by the number of correct classifications and the number of misclassifications in the experimental samples. In the confusion matrix, the overall classification accuracy (OA) and Kappa coefficient are mainly used to verify the classification results and evaluate the accuracy $[23,29]$.

The overall accuracy represents the probability of the number of samples that are correctly classified in the overall sample size and can reflect the overall accuracy of the classification results. As shown in

$$
\mathrm{OA}=\frac{\sum_{i=1}^{q} n_{i i}}{n} \times 100 \%
$$

where $\mathrm{OA}$ is the total classification accuracy, $q$ is the number of classification categories, $n$ is the total number of samples, and $n_{i i}$ is the number of discriminative samples of the $i$ th category.

The Kappa coefficient is the accuracy coefficient of the overall classification. It is a kind of ratio, which represents the ratio of error reduction between classification and completely random classification, as shown in

$$
\text { Kappa }=\frac{n \sum_{i=1}^{q} n_{i i}-\sum_{i=1}^{q}\left(n_{i+} \times n_{+i}\right)}{n^{2}-\sum_{i=1}^{q}\left(n_{i+} \times n_{+i}\right)} \times 100 \%,
$$

where $q$ is the number of classes, $n$ represents the total number of considered pixel, $n_{i i}$ is the diagonal element of the confusion matrix, $n_{i+}$ represents the marginal sum of the rows in the confusion matrix, and $n_{+i}$ represents the marginal sum of the columns in the confusion matrix.

3.2. Fishnet Model. The Fishnet model refers to the grid division of the research area based on GIS technology, where the grid scale is used as the evaluation unit to analyze the ecological security status of a selected area $[30,31]$. With the continuous advancement of science and technology and the deepening of ecological safety research, the advantages of this method have become more and more obvious: it can solve the problems of data processing accuracy in space. As the smallest analysis unit is selected as a grid, the method facilitates the effective collection of relevant data and breaks the drawbacks of using only an administrative division unit as a research unit. In addition, grid division technology of the Fishnet model can treat each grid as an independent unit by establishing a grid and then calculate each grid through the establishment of an evaluation system.

In order to better reflect the landscape ecological security status of the study area, we used the grid tool in ArcGIS to divide the study area into 307 grids of $2 \mathrm{~km} \times 2 \mathrm{~km}$ (shown in Figure 2) to break the previous inherent pattern. The division of the grid provides a more reasonable scale for the assessment of spatial change of landscape ecological security [31]. After the grid is identified, a grid layer is used to batch 


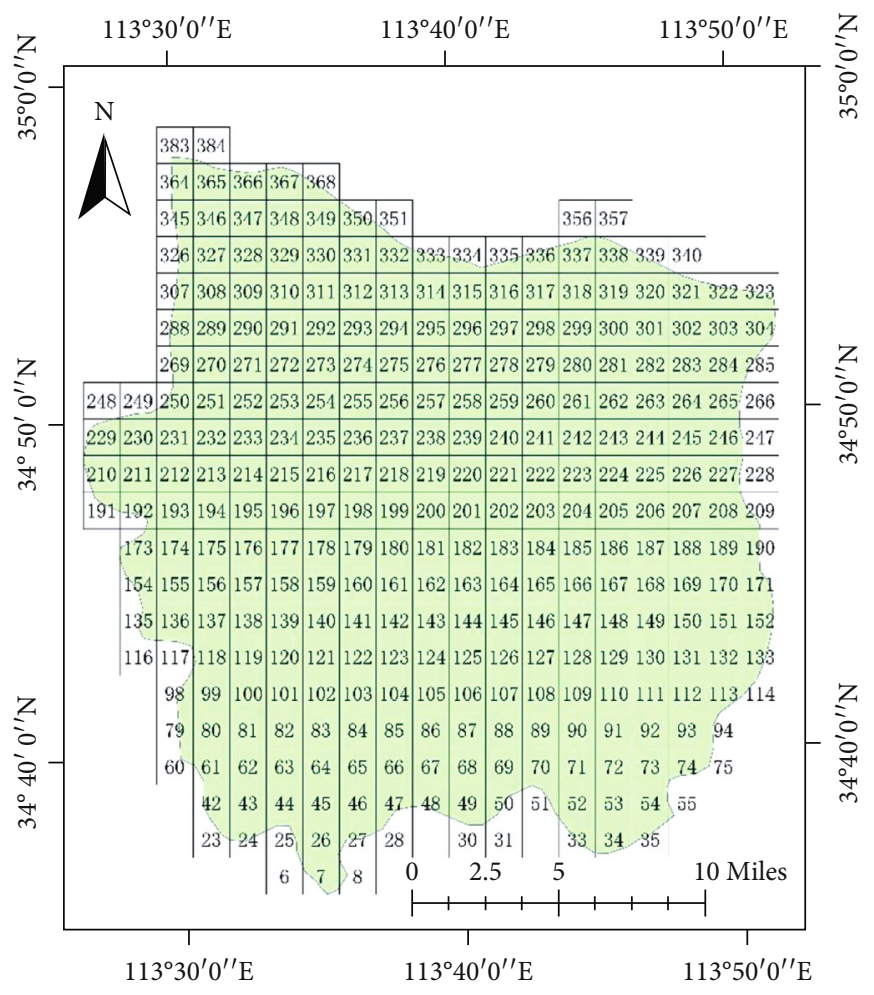

FIgURE 2: Fishnet grid division.

crop the land use classification data in each period to obtain corresponding grids. Land use and land cover maps of the small units are imported into the landscape pattern analysis software fragstats4.2 in batches mode for analysis. Then, landscape metrics of each grid in the study area can be obtained through calculation, and the calculated landscape metrics are used to construct the landscape ecological security index. Finally, the grid data is converted into a grid point file (located at the center of each grid), and the calculated landscape ecological safety index is assigned to the grid point file. During this process, spatial resolution is setting to 30 meters with the kriging interpolation method.

\section{Landscape Ecological Model}

4.1. Analysis of Landscape Pattern Changes. To analyze the changes of land use land cover landscape pattern, the ecological significance of various landscape pattern metrics in this study area was calculated [20]. By literature review [32, 33], landscape pattern can be divided into (1) patch level, which reflects the structural characteristics of a single patch in the landscape and is also the basis for calculating other landscape level indicators; (2) class level, which reflects the respective structural characteristics of different patch types in the landscape; (3) landscape level, which reflects the overall structural characteristics of the landscape. According to these landscape pattern metrics, the landscape of the study area was analyzed from a global perspective. In order to explore the structural characteristics and evolution rule of landscape pattern in various periods in Zhengzhou city, representative indicators (shown in Table 1) are selected at class level and landscape level with fully understanding the ecological significance of each landscape pattern index and the focus of the landscape structure reflected.

\subsection{Analysis of Landscape Ecological Security}

4.2.1. Landscape Disturbance Index. The landscape disturbance index describes the degree to which different landscape ecological environments are affected by natural and human activity factors. Its mathematical model can be described as

$$
W_{i}=a C_{i}+b S_{i}+c D_{i}
$$

where $W_{i}$ is the landscape disturbance index; $a, b$, and $c$ are the landscape fragmentation index, landscape separation index, and landscape dominance index, respectively; $a, b$, and $c$ represent the weight value of constructing fragmentation, separation, and dominance index, respectively. According to previous research results, and referring to the expert scoring method, the three weights are assigned as $a=0.5, b=0.3$, and $c=0.2$.

Within this model, the mathematical expression of the landscape fragmentation index $C_{i}$ is shown as

$$
C_{i}=\frac{N_{i}}{A_{i}},
$$

where $N_{i}$ is the number of landscape patches of type $i$ and $A_{i}$ is the total area of landscape patches of type $i$. 
TABLE 1: Selected landscape metrics.

\begin{tabular}{|c|c|}
\hline $\begin{array}{l}\text { Landscape } \\
\text { index }\end{array}$ & Meaning and value range \\
\hline PLAND & The proportion of each patch area to the total area $(0,100][19,34]$ \\
\hline LPI & $\begin{array}{l}\text { The largest patch area accounts for the proportion of the total area. The change of this value reflects the strength of human } \\
\text { activities. It determines the abundance of dominant species in the landscape and helps determine dominant types }(0,100][35]\end{array}$ \\
\hline PAFRAC & Reflect the complexity of the shape of the landscape type on a specific scale $[1,2][36]$ \\
\hline LSI & The degree of aggregation of patch shape features, indicating the shape of landscape [37] \\
\hline CONTAG & The aggregation degree or spreading trend of different patch types in the landscape $(0,100][38]$ \\
\hline IJI & Certain types of landscape adjacent to landscape types $(0,100][39]$ \\
\hline SHDI & $\begin{array}{l}\text { The degree of diversification of landscape types indicates the heterogeneity of the landscape and is more sensitive to the } \\
\text { uneven distribution of various patch types, SHDI equal or greater than } 0[36,40]\end{array}$ \\
\hline SHEI & There are no dominant types in the landscape, and the types are evenly distributed, indicating landscape diversity $[0,1][34]$ \\
\hline
\end{tabular}

The mathematical expression of the landscape separation index $S_{i}$ is shown as

$$
\begin{gathered}
S_{i}=\frac{1}{2} \sqrt{\frac{C_{i}}{P_{i}},} \\
P_{i}=\frac{A_{i}}{A},
\end{gathered}
$$

where $P_{i}$ is the relative coverage of the $i$ landscape and $A$ is the total landscape area.

The mathematical expression of the landscape dominance index is shown as

$$
\begin{aligned}
D_{i} & =d L_{i}+e P_{i}, \\
L_{i} & =\frac{N_{i}}{N},
\end{aligned}
$$

where $L_{i}$ is the relative density of the landscape. Related studies believe that the relative coverage has a greater contribution to the dominance of the landscape than the relative density, so the weights $d$ and $e$ are assigned values of 0.6 and 0.4.

4.2.2. Landscape Vulnerability Index. Different landscape plays different roles in the ecosystem and has different resistance to external pressure and sensitivity to external changes at the meantime. In research, this difference is usually associated with the vulnerability of the landscape, combined with previous research results [41]; on the basis of the actual situation of Zhengzhou city, values were assigned to corresponding land use types. For instance, unused land has the strongest vulnerability, and unused land is 5; the fragility of water is less than unused land, and water is set as 4; construction land is relatively stable which is set as - 1 ; and the vulnerability index of cultivated land and vegetation are 3 and 2, respectively. Due to the different dimensions of each index, the normalization of the assignment of land use type is used as the vulnerability index of this type of landscape. The calculation method is shown as

$$
V_{i}=\frac{L_{i}}{\sum_{i=1}^{5} L_{i}},
$$

where $L_{i}$ represents the score assigned to the $i$ type of land use and $V_{i}$ represents the landscape vulnerability of the $i$ type of land use.

4.2.3. Landscape Loss Index. The two variables of the landscape disturbance index and vulnerability index are superimposed and integrated to reflect the degree of loss of natural attributes of a certain landscape type after external disturbance. The calculation method is shown as

$$
R_{i}=W_{i} \times V_{i},
$$

where the calculation method and meaning of $W_{i}$ and $V_{i}$ are landscape disturbance index and landscape vulnerability index, respectively.

4.2.4. Landscape Safety Index. The landscape safety index is expressed using two variables, the landscape loss index and the landscape area. The calculation method is shown in

$$
\mathrm{LES}_{e}=\sum_{i=1}^{m} \frac{A_{e i}}{A_{e}}\left(1-10 \times R_{i}\right)
$$

where $\mathrm{LES}_{e}$ represents the landscape ecological security value of the $e$ th sampling area, $e$ is the number of grids, $A_{e}$ is the area of the eth grid, $A_{e i}$ is the area of the $i$ th landscape in the eth sampling area, and $m$ is the number of land use types.

4.3. CA-Markov Model. The Markov model is a long-term prediction method based on situation and probability of a certain event at a certain moment, using the Markov chain, to predict its changes at various moments (or periods) in the future [42]. The CA model [43] has a capacity for spatial and temporal change simulation which can be defined in equation (10). The key factor of the Markov model is determining the transition probability of an event. The main components of the cellular automata model (CA) are units, states, rules, and neighborhoods. Each unit has one of a finite number of states $[5,42,44]$. The state of the unit at a certain moment depends on the current and previous moments. All unit states can be updated at the same time according to the transition rules. The principle is that the state of a cell at the next moment is a function of its neighborhood state 

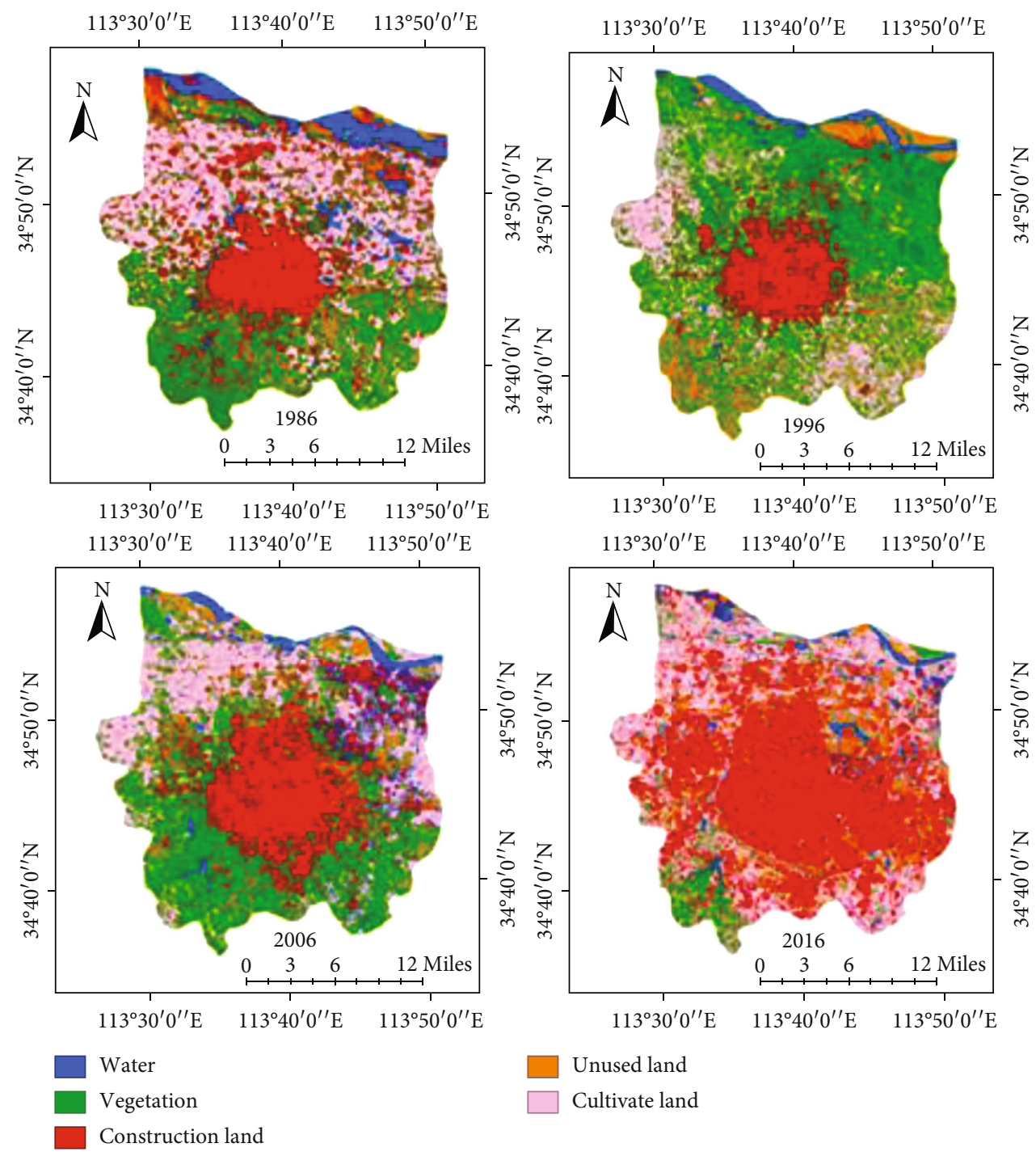

\section{Unused land}

Cultivate land

FIgUre 3: LULC classification results of Zhengzhou city from 1986 to 2016.

at the previous moment. The state variables are closely connected with the spatial position and have the concept of space and can be simulated. Complex changes in the spatial pattern.

$$
S_{(t+1)}=f\left(S_{(t)}, N\right)
$$

where $S_{(t+1)}$ and $S_{(t)}$ are the set of cell states at $t+1$ and $t$, respectively; $f$ is the transition rule function of the state of the space cell; $N$ is the cell field. $t$ and $t+1$ represent start and end times. The Markov model [45] has been widely used for trend simulation at various scenarios and can be expressed as

$$
S_{(t+1)}=P_{i j} \times S_{(t)},
$$

TABLE 2: LULC classification accuracy.

\begin{tabular}{lcccc}
\hline Parameter & 1986 year & 1996 year & 2006 year & 2016 year \\
\hline OA & $88.14 \%$ & $81.16 \%$ & $87.67 \%$ & $88.79 \%$ \\
Kappa & 0.85 & 0.75 & 0.84 & 0.86 \\
\hline
\end{tabular}

where $S_{(t+1)}$ and $S_{(t)}$ are the status at time $t+1$ and $t$, respectively.

$P_{i j}=\left(\begin{array}{ccc}P_{11} & \cdots & P_{1 n} \\ \vdots & \ddots & \vdots \\ P_{n 1} & \cdots & P_{n n}\end{array}\right),\left(0 \leq P_{i j}<1, \sum_{j=1}^{n} P_{i j}=1(i, j=1,2, \cdots, n)\right.$.

$P_{i j}$ is the transition probability matrix in a state. The CA-Markov model is a combination of the CA model and the Markov model, both of which are dynamic models 


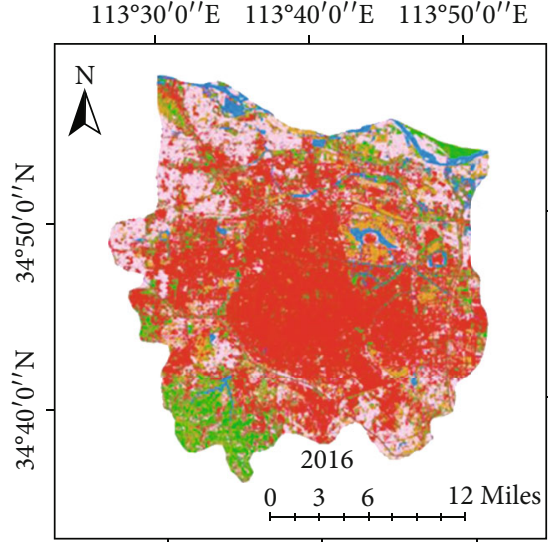

$113^{\circ} 30^{\prime} 0^{\prime \prime} \mathrm{E} \quad 113^{\circ} 40^{\prime} 0^{\prime \prime} \mathrm{E} \quad 113^{\circ} 50^{\prime} 0^{\prime \prime} \mathrm{E}$
Water
Vegetation
Construction land

$113^{\circ} 30^{\prime} 0^{\prime \prime} \mathrm{E} \quad 113^{\circ} 40^{\prime} 0^{\prime \prime} \mathrm{E} \quad 113^{\circ} 50^{\prime} 0^{\prime \prime} \mathrm{E}$

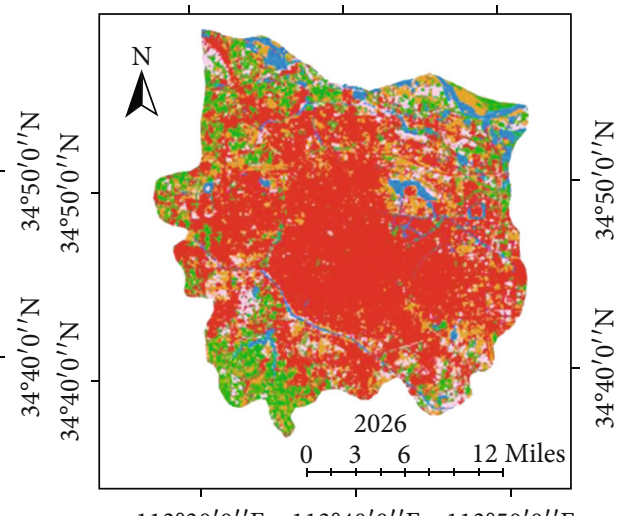

$113^{\circ} 30^{\prime} 0^{\prime \prime} \mathrm{E} \quad 113^{\circ} 40^{\prime} 0^{\prime \prime} \mathrm{E} \quad 113^{\circ} 50^{\prime} 0^{\prime \prime} \mathrm{E}$

Unused land

Cultivate land

Figure 4: LULC predicted result in 2016 and 2026 with the CA-Markov model.

TABle 3: Prediction accuracy of the CA-Markov model.

\begin{tabular}{lcccc}
\hline LULC types & 2016 status/ha & 2016 forecast/ha & Difference/ha & 79.07 \\
Water & 3993.39 & 4072.46 & -159.64 & 0.0198 \\
Construction land & 40932.9 & 40773.26 & 1126.04 & -0.0039 \\
Unused land & 15114.69 & 16240.73 & -620.84 & 0.0745 \\
Cultivated land & 36736.2 & 36115.36 & 26.46 & -0.0169 \\
Vegetation & 8268.03 & 8294.49 & 0.0032 \\
\hline
\end{tabular}

with discrete states [46]. And when combined with the CA model, the CA-Markov model can overcome the limitation that the Markov model failed to catch the spatial distribution in the future and can be used to simulate spatiotemporal changes. In this research, LULC maps obtained with RF in 1986, 1996, 2006, and 2016 were selected as the study years to calculate the transition area matrix using the Markov model. And a standard 5 by 5 contiguity filter was selected for the CA model which means the condition of future pixel is not only decided by information from previous state but also considered by corresponding surrounding pixels.

\section{Results and Discussion}

5.1. LULC Classification Maps with Random Forest. In this research, training samples were selected by an expert. And the separability of samples was also checked to verify which is greater than 1.8 to meet the basic requirements for classification. The selected four RS images of Zhengzhou city were input to the RF classifier model to obtain classification maps of corresponding date over Zhengzhou city (Figure 3). Verification samples were selected from Google Earth to verify the accuracy of the classification results. And the total classification accuracy (OA) and Kappa coefficient were assessed with the help of selected ground truth. The total classification accuracy of the four images is greater than $80 \%$ (more details can be found in Table 2) which meet the demand of further process such as landscape pattern analysis.

5.2. Predicted Result with the CA-Markov Model. In this research, with the help of the IDRISI package, LULC results of 1996, 2006, and 2016 were selected to test and verify the prediction ability of the CA-Markov model. Firstly, the transition probability matrix and transition area matrix were calculated with images for adjacent years. And next, the transferred conditional probability image was established as the land use type transition suitability image. Then, CA filter and the number of cycles were determined. Since the image interval of each phase of this research is 10 years, the number of cycles is setting as 10 , which means on the basis of LULC map of 2016 as initial to predict LULC status of 2026. Furthermore, in order to verify the reliability of the CAMarkov model, we simulated the LULC map of 2016 (shown in Figure 4) based on LULC maps of 1996 and 2006 and then calculated the accuracy of the prediction result using actually LULC maps of 2016 (Table 3). The results showed that the relative error between the predicted result and the classification result in 2016 meets the accuracy requirements. So it is scientific and reasonable to use the CA-Markov model to predict land cover in the future of the research area. So LULC map of 2026 (shown in Figure 4) was predicted with the help of the CA-Markov model and LULC results of 2006 and 2016. 
TABLE 4: Class level landscape metrics.

\begin{tabular}{|c|c|c|c|c|c|c|}
\hline Class & Year & PLAND & LPI & LSI & PAFRAC & IJI \\
\hline \multirow{5}{*}{ Water } & 1986 & 7.629 & 2.7778 & 32.2845 & 1.3375 & 83.5295 \\
\hline & 1996 & 5.8337 & 1.0263 & 63.1318 & 1.4078 & 67.0117 \\
\hline & 2006 & 5.9512 & 1.2004 & 65.9779 & 1.4327 & 75.9909 \\
\hline & 2016 & 4.6816 & 0.4844 & 55.5877 & 1.4377 & 78.8492 \\
\hline & 2026 & 4.8616 & 0.6097 & 39.9916 & 1.3333 & 73.6292 \\
\hline \multirow{5}{*}{ Built-up } & 1986 & 20.35 & 9.34 & 122.2625 & 1.5138 & 53.8871 \\
\hline & 1996 & 21.6713 & 8.4205 & 73.6836 & 1.4672 & 55.1109 \\
\hline & 2006 & 22.8781 & 14.3014 & 98.2609 & 1.4715 & 80.964 \\
\hline & 2016 & 38.96 & 30.4413 & 126.3306 & 1.4588 & 83.5898 \\
\hline & 2026 & 55.0301 & 50.4739 & 131.2537 & 1.4851 & 91.8154 \\
\hline \multirow{5}{*}{ Unused } & 1986 & 9.6823 & 0.4096 & 91.3087 & 1.384 & 76.3188 \\
\hline & 1996 & 9.023 & 1.0881 & 73.85 & 1.3822 & 43.5739 \\
\hline & 2006 & 10.5624 & 0.3 & 86.8323 & 1.3848 & 69.1466 \\
\hline & 2016 & 13.7287 & 0.9775 & 83.5122 & 1.4838 & 55.2866 \\
\hline & 2026 & 14.0307 & 0.3944 & 75.2457 & 1.5261 & 53.4874 \\
\hline \multirow{5}{*}{ Farmland } & 1986 & 30.1118 & 13.0308 & 57.1028 & 1.3251 & 71.4841 \\
\hline & 1996 & 19.9297 & 3.2892 & 121.4934 & 1.4745 & 76.2108 \\
\hline & 2006 & 24.387 & 7.9274 & 99.8876 & 1.4846 & 83.589 \\
\hline & 2016 & 34.7818 & 7.2556 & 122.6385 & 1.4875 & 86.2797 \\
\hline & 2026 & 10.0261 & 6.819 & 113.981 & 1.5407 & 85.0774 \\
\hline \multirow{5}{*}{ Vegetation } & 1986 & 32.231 & 2.8358 & 167.5532 & 1.5353 & 83.4216 \\
\hline & 1996 & 43.543 & 30.0522 & 166.4137 & 1.5291 & 88.8913 \\
\hline & 2006 & 36.224 & 4.3383 & 147.6667 & 1.5098 & 86.2738 \\
\hline & 2016 & 7.852 & 0.029 & 126.5385 & 1.5045 & 67.2062 \\
\hline & 2026 & 16.0515 & 1.28 & 135.1132 & 1.3641 & 65.2682 \\
\hline
\end{tabular}

\subsection{Results of Landscape Pattern}

5.3.1. Changes of Landscape Pattern in Class Level. Based on the obtained land cover classification results of each period, class level landscape pattern indexes were obtained with the help of fragstats4.2 (shown in Table 4). Results of the PLAND index showed that the dominated land cover types are farmland and built-up area in Zhengzhou city. In the period of 1986, the rank of PLAND in descending order is vegetation, farmland, built-up area, unused land, and water body. While in the period of 2016, the maximum PLAND index is builtup area and followed by farmland. The cause of these changes can be ascribed to two aspects. One is that, as the core area of national food production, governments of Henan province at all levels and the Department of Land and Resources have comprehensively strengthened the goal of arable land protection, which has further promoted the conservation and intensive use of land. The other one is that developing the economy, various provinces and cities in Henan have accelerated reclamation and the same time high-standard farmland construction to ensure the amount of arable land; on the other hand, the rapid economic development in the urban area of Zhengzhou has resulted in continuous urban construction land expand. The PLAND index of vegetation has changed significantly, from 32.231 in 1986 to 7.852 in
TABLE 5: Landscape level metrics.

\begin{tabular}{ccccc}
\hline Year & CONTAG & IJI & SHDI & SHEI \\
\hline 1986 & 31.0152 & 81.8333 & 1.6863 & 0.9412 \\
1996 & 33.2598 & 76.9566 & 1.5993 & 0.8926 \\
2006 & 35.3083 & 74.9736 & 1.7025 & 0.9502 \\
2016 & 38.2084 & 74.9651 & 1.3881 & 0.7747 \\
2026 & 45.262 & 82.8463 & 1.4655 & 0.7925 \\
\hline
\end{tabular}

2016, indicating that this type of land use type accounts for a large reduction in the overall landscape area.

Particularly, by prediction in 2026, there will be almost no changes in the area of water and unused land; the PLAND value of vegetation has increased, the area occupied by builtup area has further increased, and the PLAND index of farmland has decreased significantly. This result indicates regional urbanization and industrialization. During the process, the farmland was severely disturbed by human activities, and the farmland was squeezed by large-scale development activities by humans.

For the LPI index, the value of water has decreased greatly from 2.8 in 1986 to 0.48 in 2016 , which indicated that the largest patch area of the water area has decreased, 

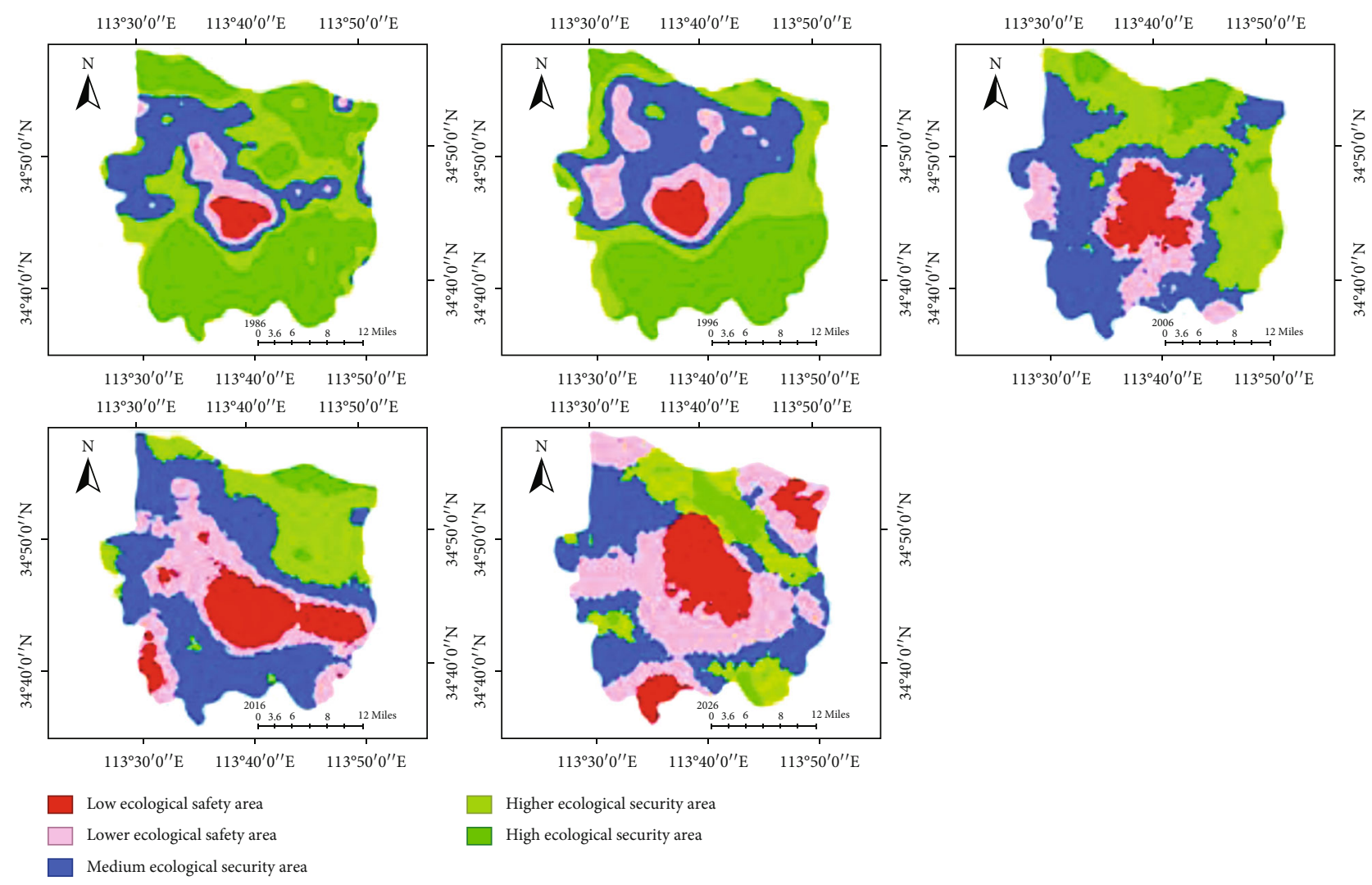

$113^{\circ} 30^{\prime} 0^{\prime \prime} \mathrm{E} \quad 113^{\circ} 40^{\prime} 0^{\prime \prime} \mathrm{E} \quad 113^{\circ} 50^{\prime} 0^{\prime \prime} \mathrm{E}$

Higher ecological security area

High ecological security area

FIgURE 5: Ecological security distribution maps.

gradually shrinking, and the distribution is gradually fragmented; the LPI index of vegetation was 0.029 by 2016; this showed that the maximum patch area of vegetation first increases and then decreases, and its fragmentation degree first relaxes and then increases year by year. In addition, the LPI index value of built-up area increased greatly during the period from 1986 to 2016, which to a certain extent reflects that the urban construction of Zhengzhou city is more concentrated and the area of urban contiguous areas has increased significantly. The LPI index of farmland decreased from 13.03 in 1986 to 3.29 in 1996 and then increased to 7.26 in 2016, indicating that the largest patch area of farmland is fluctuating. According to the prediction results, the increase in the LPI value of water and vegetation indicates that the degree of fragmentation has decreased; the maximum patch index of unused land and farmland has decreased, and the distribution of farmland has become more concentrated.

From the perspective of the LSI index, the value of water is generally lower than that of other land types, mainly because its spatial distribution is relatively scattered, and the degree of aggregation is lower than that of other land types. During the study period, the PAFRAC index of various land use types in the urban area of Zhengzhou showed that vegetation was always the landscape with the largest PAFRAC index, indicating that the shape of vegetation in such patches was the most complicated. The PAFRAC index of built-up land has declined in fluctuations, indicating that the shape of this landscape type tends to be simple, and the

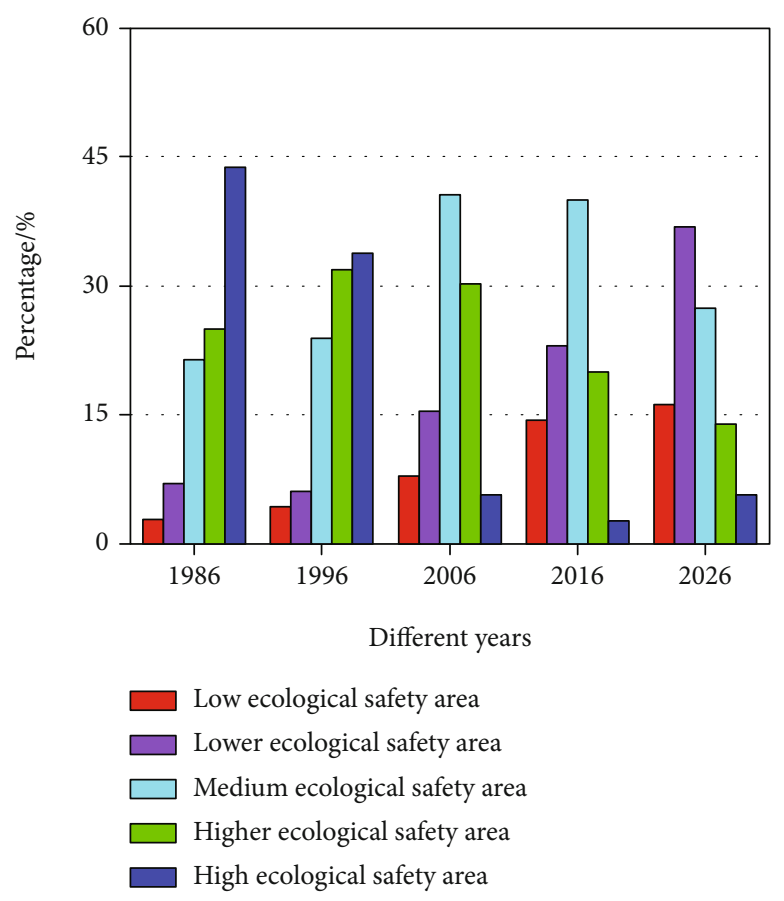

FIgURE 6: Percentage of ecological security levels in different years.

complexity is reduced. The PAFRAC of water, farmland, and unused land have changed, showing an increasing trend, indicating that the shapes of these three landscape types are 
TABLe 6: Percentage of ecological security level.

\begin{tabular}{|c|c|c|c|c|c|}
\hline \multirow{2}{*}{ Ecological security level } & \multicolumn{5}{|c|}{ Percentage of each level (\%) } \\
\hline & 1986 & 1996 & 2006 & 2016 & 2026 \\
\hline Low ecological security area & 2.80 & 4.32 & 7.96 & 14.36 & 16.17 \\
\hline Lower ecological security area & 6.98 & 6.08 & 15.48 & 22.98 & 36.88 \\
\hline Medium ecological security area & 21.40 & 23.88 & 40.55 & 40.03 & 27.36 \\
\hline Higher ecological security area & 24.97 & 31.96 & 30.26 & 19.95 & 13.90 \\
\hline High ecological security area & 43.85 & 33.76 & 5.74 & 2.68 & 5.69 \\
\hline
\end{tabular}

becoming more complicated. It is estimated by 2026, the LSI index of land use types other than built-up land and vegetation will decrease, indicating that the degree of aggregation is reduced; the PAFRAC of unused land and farmland has increased, indicating that the complexity of patch shape has increased, and its shape has become more and more irregular.

In terms of the IJI index, vegetation and unused land showed a downward trend, indicating that the adjacent landscape types of these two land types decreased year by year. The IJI index value of water decreased from 83.53 in 1986 to 67.01 in 1996 and increased to 78.85 in 2016 and then became relatively stable. By 2016, the IJI index of built-up land increased significantly compared to 1986, mainly due to the mutual transformation of land types around built-up land and the increase in the types of adjacent landscapes. It is estimated that by 2026 , compared with 2016, the spread of land use types other than built-up land and the parallel index will change slightly, indicating that the adjacent patch types of land types other than built-up land have decreased.

5.3.2. Changes of Landscape Index Landscape Level. From the analysis of the landscape pattern in the landscape level, the calculation results of landscape metrics in different periods of Zhengzhou city are shown in Table 5.

The CONTAG index of Zhengzhou city increased from 31.02 in 1986 to 38.21 in 2016. It can be seen that the concentration of different landscape types in the study area has increased. During the study period, the IJI index of Zhengzhou city showed a decreasing trend, from 81.84 in 1986 to 74.97 in 2006, which indicated that the number of adjacent patches of a landscape type has decreased. However, the probability of all patch types adjacent to each other has stabilized after 2006. In terms of the Shannon diversity index (SHDI), whose value decreased in fluctuation, it indicated that the landscape types in the study area are gradually decreasing and the landscape diversity has decreased. In terms of the SHEI index, the uniformity of the distribution of various landscape types showed a decreasing-increasingdecreasing trend, which decreased from 0.94 in 1986 to 0.89 in 1996 and then increased to 0.95 in 2006. And SHEI increased to 0.7747 in 2016, which indicated that there were no particularly obvious advantages in the landscape in 1986, 1996, and 2006, while the advantages of landscape were obvious in 2016. According to the predicted results in 2026, SHDI and SHEI of the study area will be increased, which indicated that the intensity of human disturbance to the landscape has increased. And the predicted result also showed that the intensity of human disturbance to the landscape will increase, the diversity of the landscape will increase, and the control effect of the landscape dominance category on the overall landscape will be weakened, different land use types are more concentrated, and the types of adjacent features were increased.

5.4. Landscape Ecological Security Analysis. Due to the characteristics of spatial heterogeneity in ecological security assessment, in this study, the Fishnet tool was used to refine the units of the ecological security assessment. By calculating the corresponding landscape index in the constructed ecological security assessment model, the landscape ecological security index value of each grid is obtained, and this value is interpolated to the center of the grid using the kriging interpolation method to obtain the ecological security distribution maps.

Furthermore, according to the natural discontinuity classification method [47], the ecological security of the study area is divided into five levels: low ecological safety area, lower ecological safety area, medium ecological safety area, higher ecological safety area, and high ecological safety area (Figure 5).

From the characteristics of spatial distribution of LULC types, the high, higher, and medium security areas are mainly distributed in vegetation land cover area in the north and south, and the low and lower safety areas are distributed in the central built-up area and unused land areas.

By quantitative analysis (Figure 6 and Table 6), in 1986, the ecological security of Zhengzhou city was dominated by high-safety, higher-safety, and medium-safety areas, and the low-security zones were less distributed, indicating that the overall ecological security level of Zhengzhou city during that period was relatively high. Compared ecological security of 1996 with 1986, the medium and higher ecological security area status of ecological security in 1996 increased, while lower and low ecological security areas are almost stable. And the location of the low ecological safety zone has hardly changed when compared with 1986, and it is roughly in the center of Zhengzhou city. Compared with the ecological of 2006 to 1996, the lower and medium ecological safety zones increased significantly in 2006, and the medium ecological safety zones were widely distributed, and the high ecological safety zones decreased. This was mainly because with the rapid development of urbanization, the urban construction land expanded greatly; by 2016, the area of urban construction land will reach to $40 \%$, frequent human activities have intensified the degree of land use, and ecological security is in a low state. Compared ecological security of 2016 with 
2006, most areas are still in a state of low ecological security. The most obvious area is still located in the middle of Zhengzhou city, indicating that the urbanization of Zhengzhou city is developing rapidly, the population is growing rapidly, and human activities are frequent. Severe expansion has put a lot of pressure on the regional ecological environment, leading to a decrease in its ecological security. It is predicted that by 2026, the proportion of lower ecological safety areas will be the largest. Compared with 2016, the area of low ecological safety areas in this region has not increased much, the medium ecological safety areas have dropped by about $10 \%$, and the percentage of higher ecological safety areas has declined, but the proportion of high ecological safety areas has increased slightly.

\section{Conclusion}

In this research, we have proposed an ecological security assessment framework based on random forest algorithm, Fishnet model, landscape ecology model, and CA-Markov model. With the support of multitemporal remote sensing and GIS technology, ecological security of research area in 1986, 1996, 2006, 2016, and for predicted 2026 were calculated and simulated. The research results showed that due to the significant urbanization process and certain problems in the economic development model, the largest patch area of the water has been significantly reduced, from 2.78 to 0.48 . The fragmentation of water is increasing year by year. However, the construction land is more concentrated, the area of urban contiguous areas has increased significantly, and the spread and juxtaposition index value of construction land has increased from 53.8871 to 83.5898 . It is predicted that by 2026, the shape of unused land and farmland will become more irregular, and the area of construction land will further increase and the distribution will be more concentrated. The results of landscape ecological security evaluation showed that between 1986 and 1996, the dominated security level is high grade, while between 2006 and 2016, the area of medium ecological security zone was in major. It is predicted that the landscape ecological security situation of Zhengzhou city will continue to decline in 2026 , but the percentage of high ecological security areas will increase slightly.

The research results can provide basic information and decision support for government agencies and land use planners to ensure responsible and sustainable development of the urban environment and ecology. And the novelty of this paper is in the direction of successfully implementing the landscape ecology model for urban security assessment.

\section{Data Availability}

Researchers who are interested in this code can download it from hyperlink https://pan.baidu.com/s/ 1YeGDwd4DoOEkZpOh4vswKg, using password xdrg, using password i98q, or contact the corresponding author to obtain source data to conduct secondary analysis.

\section{Disclosure}

Pei Liu's current address is No. 2001 Shiji Road, Jiaozuo, Henan, China.

\section{Conflicts of Interest}

The authors declare that they have no conflicts of interest.

\section{Authors' Contributions}

P.L. contributed to the conceptualization, methodology, formal analysis, writing and preparing the original draft, and project administration; P.L., C.M., and HB. Z. wrote, reviewed, and edited the manuscript; XY. Z. and XF. L. performed the experiment. All authors contributed equally to this work.

\section{Acknowledgments}

This research was funded by the National Natural Science Foundation of China Grant number 41601450, the Outstanding Youth fund of Henan Polytechnic University Grant number J2021-3, the Key Technology R and D Program of Henan Province (No. 182102310860), and the China Scholarship Council Grant number 201808410212.

\section{References}

[1] D. Liu and Q. Chang, "Ecological security research progress in China," Acta Ecologica Sinica, vol. 35, no. 5, pp. 111-121, 2015.

[2] J. Wu, "Key concepts and research topics in landscape ecology revisited: 30 years after the Allerton Park workshop," Landscape Ecology, vol. 28, no. 1, pp. 1-11, 2013.

[3] X. Liang, X. Jin, J. Ren, Z. Gu, and Y. Zhou, "A research framework of land use transition in Suzhou city coupled with land use structure and landscape multifunctionality," Science of the Total Environment, vol. 737, article 139932, 2020.

[4] X. Yuantao, M. Qingwen, Y. Zheng et al., "Identifying landscape pattern metrics for the Hani terrace in Yunnan, China," Journal of Resources and Ecology, vol. 4, no. 3, pp. 212-219, 2013.

[5] Z. K. Motlagh, A. Lotfi, S. Pourmanafi, S. Ahmadizadeh, and A. Soffianian, "Spatial modeling of land-use change in a rapidly urbanizing landscape in Central Iran: integration of remote sensing, CA-Markov, and landscape metrics," Environmental Monitoring and Assessment, vol. 192, no. 11, p. 695, 2020.

[6] C. Zhu, X. Zhang, M. Zhou et al., "Impacts of urbanization and landscape pattern on habitat quality using OLS and GWR models in Hangzhou, China," Ecological Indicators, vol. 117, article 106654, 2020

[7] W. Gao, G. Cheng, and C. Liu, "Incorporating carbon sink of harvested wood products into ecological footprint accounting: model and case study," Ecosystem Health and Sustainability, vol. 6, no. 1, article 1770629, 2020.

[8] Q. Yang, G. Liu, Y. Hao et al., "Quantitative analysis of the dynamic changes of ecological security in the provinces of China through emergy-ecological footprint hybrid indicators," Journal of Cleaner Production, vol. 184, pp. 678-695, 2018. 
[9] Z. T. Li, M. Li, and B. C. Xia, "Spatio-temporal dynamics of ecological security pattern of the Pearl River Delta urban agglomeration based on LUCC simulation," Ecological Indicators, vol. 114, article 106319, 2020.

[10] J. Wu, X. Wang, B. Zhong et al., "Ecological environment assessment for Greater Mekong Subregion based on pressure-state-response framework by remote sensing," Ecological Indicators, vol. 117, article 106521, 2020.

[11] Y. Fan and C. Fang, "Evolution process and obstacle factors of ecological security in western China, a case study of Qinghai province," Ecological Indicators, vol. 117, article 106659, 2020.

[12] S. Wei, J. Pan, and X. Liu, "Landscape ecological safety assessment and landscape pattern optimization in arid inland river basin: take Ganzhou District as an example," Human and Ecological Risk Assessment, vol. 26, no. 3, pp. 782-806, 2020.

[13] B. Sun, J. Tang, D. Yu, Z. Song, and P. Wang, "Ecosystem health assessment: a PSR analysis combining AHP and FCE methods for Jiaozhou Bay, China," Ocean \& Coastal Management, vol. 168, pp. 41-50, 2019.

[14] J. Peng, M. Zong, Y. Hu, Y. Liu, and J. Wu, “Assessing landscape ecological risk in a mining city: a case study in Liaoyuan City, China," Sustainability, vol. 7, no. 7, pp. 8312-8334, 2015.

[15] P. Liang, D. Liming, and Y. Guijie, "Ecological security assessment of Beijing based on PSR model," Procedia Environmental Sciences, vol. 2, pp. 832-841, 2010.

[16] T. Weber, "Landscape ecological assessment of the Chesapeake Bay watershed," Environmental Monitoring and Assessment, vol. 94, no. 1-3, pp. 39-53, 2004.

[17] G. Wang and W. Wu, "Assessment on ecological security spatial differences of west areas of Liaohe River based on GIS," Huan jing ke xue= Huanjing kexue, vol. 26, no. 5, pp. 28-33, 2005.

[18] D. Pan, H. Jia, and Y. Yuan, “A GIS-based ecological safety assessment of Wushen Banner, China," Human and Ecological Risk Assessment, vol. 21, no. 2, pp. 297-306, 2015.

[19] P. Parivar, D. Quanrud, A. Sotoudeh, and M. Abolhasani, "Evaluation of urban ecological sustainability in arid lands (case study: Yazd-Iran)," Environment, Development and Sustainability, vol. 23, no. 2, pp. 2797-2826, 2021.

[20] J. Zhang, M. Qu, C. Wang, J. Zhao, and Y. Cao, "Quantifying landscape pattern and ecosystem service value changes: a case study at the county level in the Chinese loess plateau," Global Ecology and Conservation, vol. 23, article e01110, 2020.

[21] B. Rasti and P. Ghamisi, "Remote sensing image classification using subspace sensor fusion," Information Fusion, vol. 64, pp. 121-130, 2020.

[22] S. Amini, S. Homayouni, A. Safari, and A. A. Darvishsefat, "Object-based classification of hyperspectral data using random forest algorithm," Geo-Spatial Information Science, vol. 21, no. 2, pp. 127-138, 2018.

[23] T. D. Acharya, A. Subedi, and D. H. Lee, "Evaluation of machine learning algorithms for surface water extraction in a Landsat 8 scene of Nepal," Sensors, vol. 19, no. 12, article 2769, 2019.

[24] P. Probst and A. L. Boulesteix, "To tune or not to tune the number of trees in random forest," The Journal of Machine Learning Research, vol. 18, pp. 6673-6690, 2017.

[25] H. Seo, M. Badiei Khuzani, V. Vasudevan et al., "Machine learning techniques for biomedical image segmentation: an overview of technical aspects and introduction to state-of-art applications," Medical Physics, vol. 47, no. 5, pp. E148-E167, 2020.
[26] H. Deng and G. Runger, "Gene selection with guided regularized random forest," Pattern Recognition, vol. 46, pp. 34833489, 2013.

[27] J. L. Speiser, M. E. Miller, J. Tooze, and E. Ip, “A comparison of random forest variable selection methods for classification prediction modeling," Expert Systems with Applications, vol. 134, pp. 93-101, 2019.

[28] P. Lou, B. Fu, H. He et al., “An optimized object-based random forest algorithm for marsh vegetation mapping using highspatial-resolution GF-1 and ZY-3 data," Remote Sensing, vol. 12, no. 8, article 1270, 2020.

[29] Y. M. Asare, E. K. Forkuo, G. Forkuor, and M. Thiel, "Evaluation of gap-filling methods for Landsat 7 ETM+ SLC-off image for LULC classification in a heterogeneous landscape of West Africa," International Journal of Remote Sensing, vol. 41, no. 7, pp. 2544-2564, 2020.

[30] P. Šímová and K. Gdulová, "Landscape indices behavior: a review of scale effects," Applied Geography, vol. 34, pp. 385394, 2012.

[31] Y. Shao, Y. Wang, and Y. Li, "Spatial-temporal response and comprehensive evaluation of ecological environment of prairie coal-electricity base development," Journal of China Coal Society, vol. 44, pp. 3874-3886, 2019.

[32] J. Kupfer, "Landscape ecology and biogeography: rethinking landscape metrics in a post-FRAGSTATS landscape," Progress in Physical Geography: Earth and Environment, vol. 36, no. 3, pp. 400-420, 2012.

[33] J. N. Inkoom, S. Frank, K. Greve, U. Walz, and C. Fürst, "Suitability of different landscape metrics for the assessments of patchy landscapes in West Africa," Ecological Indicators, vol. 85, pp. 117-127, 2018.

[34] Y. C. Weng, "Spatiotemporal changes of landscape pattern in response to urbanization," Landscape and Urban Planning, vol. 81, no. 4, pp. 341-353, 2007.

[35] Y. Song, X. Song, and G. Shao, "Effects of green space patterns on urban thermal environment at multiple spatial-temporal scales," Sustainability, vol. 12, no. 17, article 6850, 2020.

[36] H. Zhao, Z. Ren, and J. Tan, “The spatial patterns of land surface temperature and its impact factors: spatial non-stationarity and scale effects based on a geographically-weighted regression model," Sustainability, vol. 10, no. 7, article 2242, 2018.

[37] Q. Zhang, C. Chen, J. Wang et al., "The spatial granularity effect, changing landscape patterns, and suitable landscape metrics in the Three Gorges Reservoir Area, 1995-2015," Ecological Indicators, vol. 114, article 106259, 2020.

[38] D. Simsek and E. Sertel, "Spatial analysis of two different urban landscapes using satellite images and landscape metrics," Photogrammetric Engineering and Remote Sensing, vol. 84, no. 11, pp. 711-721, 2018.

[39] R. D. Bidgoli, H. Koohbanani, and M. Yazdani, "Investigation on ecosystem degradation induced by LU/LC changes using landscape pattern indices analysis," Arabian Journal of Geosciences, vol. 11, no. 16, 2018.

[40] L. Hou, F. Wu, and X. Xie, "The spatial characteristics and relationships between landscape pattern and ecosystem service value along an urban-rural gradient in Xi' an city, China," Ecological Indicators, vol. 108, article 105720, 2020.

[41] H. Xie, "Spatial characteristic analysis of land use eco-risk based on landscape structure: a case study in the Xingguo County, Jiangxi Province," China Environmental Science, vol. 31, pp. 688-695, 2011. 
[42] A. Mohamed and H. Worku, "Simulating urban land use and cover dynamics using cellular automata and Markov chain approach in Addis Ababa and the surrounding," Urban Climate, vol. 31, article 100545, 2020.

[43] S. Yan, Q. Zhixin, L. Xiaoping, N. Ning, and Z. Hui, "Urban land use and land cover classification using multisource remote sensing images and social media data," Remote Sensing, vol. 11, no. 22, article 2719, 2019.

[44] R. Qiu, W. Xu, J. Zhang, and K. Staenz, "Modeling and simulating industrial land-use evolution in Shanghai, China," Journal of Geographical Systems, vol. 20, no. 1, pp. 57-83, 2018.

[45] L. Chu, T. Sun, T. Wang, Z. Li, and C. Cai, "Evolution and prediction of landscape pattern and habitat quality based on CAMarkov and InVEST model in Hubei section of three gorges reservoir area (TGRA)," Sustainability, vol. 10, no. 11, article 3854, 2018.

[46] Y. Yu, M. Yu, L. Lin et al., "National green GDP assessment and prediction for China based on a CA-Markov land use simulation model," Sustainability, vol. 11, no. 3, p. 576, 2019.

[47] X. Xu, H. Lin, and Z. Fu, "Regional ecological risk assessment of wetland in the Huanghe River Delta," Acta Scientiarum Naturalium-Universitatis Pekinensis, vol. 37, pp. 111-120, 2001. 